Both areas were concerned about missed 'clues' from the home environment. Interestingly the nurses within the more urban, generally lower socioeconomic class area, within the Telford team, repeatedly mentioned easier, 'less distracted' reviews in a clinic environment.

In Conclusion nurse-led clinics may be a useful adjunct for hospices and the option does fit with individualised care. However we encountered significant difficulties with patient demographics and nurse-assessed suitability for this service.

\section{ACUTE PALLIATIVE INTERVENTION (API): FACILITATING ENHANCED WORKING BETWEEN THE CRITICAL CARE OUTREACH TEAM (CCOT) AND THE HOSPITAL SPECIALIST PALLIATIVE CARE TEAM (SPCT) TO ENSURE APPROPRIATE COMFORT CARE FOR THE ACUTELY ILL}

Felicity Dewhurst, Alex Nicholson, Lindsay Garcia, Isabel Gonzalez, Martin Johnson, Tony Roberts. Newcastle University, James Cook University Hospital, South Tees Hospitals NHS Foundation Trust

\subsection{6/spcare-2020-PCC.141}

Background Patients with life limiting illnesses commonly present to hospital acutely and are referred to the Critical Care Outreach Team (CCOT). It is frequently concluded that escalation of care is inappropriate due to the partially or completely irreversible nature of deterioration. In such cases escalation of palliative care is fundamental. We performed a retrospective service evaluation of patient outcome following critical care outreach review in patients deemed unsuitable for critical care admission and evaluated appropriateness of referral to the Specialist Palliative Care Team (SPCT).

Methods This was a two-centre study. Paper medical notes from a were reviewed. SPCT involvement was assessed for appropriateness using a conceptual framework.

Results 50 patients' (evaluated by CCOT but unsuitable for a higher level of care between October and December 2018) notes were reviewed. 34 (68\%) died and 16 (32\%) were discharged. Of those who died, the median time from initial CCOT review to death was 4.5 days. $6(12 \%)$ of patients were referred to the SPCT. A further 7 (14\%) were reviewed by the SPCT who proactively identified patients as end of life care through the discontinuation of physiological observations. However, 45 (90\%) should have been referred.

Conclusions CCOTs identify patients who are inappropriate for higher-level care. Most of these patients are unlikely to survive their hospital admission and the vast majority would benefit from SPCT review for appropriate symptom management and Advanced Care Planning (ACP). The term Acute Palliative Intervention (API) could be utilised to change the language and culture of care decisions.

\section{THE PREVALENCE OF FRAILTY AMONGST HOSPICE IN- PATIENT POPULATIONS: WHAT DOES THIS MEAN FOR OUR PATIENTS AND THE CARE WE DO AND DO NOT PROVIDE?}

Felicity Dewhurst, Barbara Hanratty, Paul Paes, Katie Frew, Daniel Stowe, Simon Gordon, James Ellam, Angela Egdell, Andrea Brown, Andrew Hughes, Joanna Elverson, Paul McNamara, Owen Lever. Newcastle University, St Oswald's Hospce, Hospices North East

10.1136/spcare-2020-PCC.142
Background Improved understanding of how to provide palliative care to the growing number of people living and dying with frailty is an international priority. Appropriate models of care may have similarities and differences to existing specialist palliative care (SPC) provision. Patients who currently access SPC may subjectively be described as frail; however, there is limited data on actual frailty prevalence and how frailty is associated with demographics, diagnoses and outcomes.

Methods Specific measures of frailty are not routinely recorded in SPC; therefore, measures of performance that are consistently collected were mapped to frailty level. Hospices North East (a collaborative of independent hospices) have an established dataset detailing the care they provide for whom. Analysis of this dataset established levels of frailty and its relationships.

Results The Australia-Modified Karnofsky Performance Status (AKPS) can be mapped to Rockwood's Clinical Frailty Scale to provide a proxy measure of frailty. 520 discharges or deaths (from 455 patients) occurred in three independent hospices in the Northeast of England from April 1st 2017 to March 31st 2018. Admission AKPS was available on 420 discharges or deaths (from 407 patients). On admission to the hospice the prevalence of very severe frailty (AKPS 10-20) was 26.4\%; severe frailty (AKPS 30) was 11.4\%; moderate frailty (AKPS 40-50) was 35.5\% and mild frailty (AKPS 60) was $17.6 \%$. One-off high levels of frailty and progressively increasing frailty are detrimentally related to prognosis and length of stay.

Conclusions There is a significant burden of frailty in the current hospice in-patient population. Therefore, much may be learned from contemporary service provision when considering applicable future palliative care models for those with frailty. As with current patients a two-tier in-patient model comprising of both intensive medically led short stay units and nurse led longer stay units or community beds may be useful.

\section{IS THERE A ROLE FOR CARDIOPULMONARY RESUSCITATION IN PALLIATIVE CARE? DEVELOPING A HOSPICE APPROPRIATE SERVICE IN RESPONSE TO A PATIENT CASE}

Hayley Evans, Tony Blower. St Michael's Hospice

\subsection{6/spcare-2020-PCC.143}

Background Attempting cardiopulmonary resuscitation (CPR) in the hospice setting can be a divisive issue, with some arguing it should not be attempted. Within St Michael's Hospice, we are managing an increasing number of patients earlier in their illness, when CPR may remain appropriate. Following a patient case, we reviewed our CPR service to ensure it was still meeting the needs of our patients.

Method Following the successful resuscitation of a 42-year-old patient with locally advanced pancreatic cancer after an in hospice cardiac arrest, using basic life support with automatic external defibrillation (AED), the case was brought to the monthly Significant Event Meeting (SEM). This proved a controversial case, although overall we agreed CPR was appropriate for this patient.

Results In response to the SEM, several changes were made. As part of this, a multi-professional educational 